\title{
Targeted radiotherapy for neuroblastoma
}

\section{Background}

Biologically targeted radiotherapy is an established treatment for thyrotoxicosis and thyroid cancer. ${ }^{1}$ The principle is to take advantage of the ability of the thyroid and, to a lesser degree, differentiated thyroid carcinoma to concentrate and retain iodide. In this situation the radionuclide $\left({ }^{131} \mathrm{I}\right)$ in ionic form is a natural substrate for targeting thyroid tissue, thus acting both as the vector, or 'missile', and the 'warhead'. The success of this form of radiotherapy in metastatic papillary and follicular thyroid carcinoma ${ }^{2}$ is due to the specificity of targeting and the resulting concentration and retention of ${ }^{131} \mathrm{I}$ in cells of thyroid origin, after the ablation of residual normal thyroid tissue, being sufficient to result in tumour eradication. Unfortunately the thyroid is unique and targeted radioiodine treatment for other tumours requires specific vectors to carry the 'warhead'.With the development of monoclonal antibodies in $1975^{3}$ it was hoped that Erlich's concept of an immunological magic bullet would quickly be realised, but more than 20 years later the applications of targeted monoclonal antibody treatment are distinctly limited in clinical oncology. There are several reasons for this failure of monoclonal antibodies to produce results: firstly, antigen expression on tumour cells is variable and tumour antigens are often expressed to some degree on normal cells, so the specificity is poor. Furthermore there is often poor penetration of large immune globulin molecules into tumours, a problem which has not been overcome to any significant degree by employing smaller molecular weight fragments. Furthermore murine monoclonal antibodies are themselves immunogenic and this precludes repeated administration. Despite these limitations, there have been attempts to employ targeted monoclonals in the treatment of neuroblastoma ${ }^{45}$ and regional targeting of leukaemia within the cerebrospinal fluid ${ }^{6}$ has produced responses.

The search has continued for low molecular weight compounds, which utilise metabolic pathways with high tumour specificity. Currently one of the most promising compounds is metaiodobenzylguanidine (mIBG) which exploits the active uptake pathway for noradrenaline expressed in sympathetic nervous tissues and in tumours of neural crest origin. Radioiodinated adrenergic blocking agents were originally developed as adrenomedullary imaging agents ${ }^{7}$ and the first human studies were described in adults with phaeochromocytoma ${ }^{8}$ and soon after in children with neuroblastoma. ${ }^{9}$ Laboratory studies have elucidated the mechanism of uptake of mIBG in neural crest tumours, while clinical studies have exploited the high affinity of ${ }^{131} \mathrm{I}$-mIBG for neuroblastoma in particular and targeted treatment is now moving into front line investigational protocols in Europe.

\section{Laboratory studies}

The mechanisms of uptake and retention of radioiodinated mIBG in tissues and tumours of neural crest origin have been extensively studied. ${ }^{10}$ The cellular uptake of both noradrenaline and mIBG into adrenomedullary cells and neuroblastoma takes place both by a specific uptake system, known as uptake-one, and by a non-specific mechanism, presumed to be passive diffusion. Uptake-one is an active process by the noradrenaline transport transmembrane protein and is an high affinity, saturable, sodium, energy, and temperature dependent process, which is sensitive to ouabain and competitively blocked by sympathomimetics such as imipramine. ${ }^{11}$ In contrast the non-specific uptake system is energy independent, ouabain insensitive, and unsaturable at concentrations of at least $5 \mathrm{mM}$. Not all neuroblastoma cell lines possess the active transport mechanism, but in the human SK-N-SH neuroblastoma cell line specific uptake is $>95 \%$ of total uptake at a concentration of $10 \mathrm{nM}$ and is still $80 \%$ at the saturation concentration. ${ }^{12}$ As plasma concentrations of mIBG after therapeutic doses are $<0.1 \mu \mathrm{M}$, it is likely that in the clinical setting uptake-one is the predominant uptake system for mIBG. There are important differences in the uptake and storage of mIBG between neuroblastoma and phaeochromocytoma as demonstrated by comparative studies of PC-12 (phaeochromocytoma) and SK-N-SH (neuroblastoma) cell lines: storage in PC-12 cells is predominantly in the abundant neurosecretory granules and can be depleted by reserpine. In contrast, with SK-N-SH cells, where neurosecretory cells are sparse, the uptake is in the cytoplasm. ${ }^{13}$ The potent depletion of mIBG in SK-N-SH cells by the uptake-one inhibitor, imipramine, suggests that retention of mIBG is the result of reuptake of mIBG that has passively diffused out of cells.

Pharmacological manipulation to enhance uptake and/or retention of $\mathrm{mIBG}$ in neuroblastoma cell lines has been unsuccessful with nifedipine or reserpine. Pretreatment with other agents, however, may exert an effect and interferon has been shown to improve retention in vitro. ${ }^{14} \mathrm{~A}$ recent fortuitous observation of up-regulation of the noradrenaline transporter and enhanced uptake of mIBG after cisplatin pretreatment of the neuroblastoma cell line SK-N-BE may, if confirmed in vivo, have clinical importance in the scheduling of multimodal treatment. ${ }^{15}$

The distribution of mIBG in surgically excised human neuroblastoma tumours has been studied 24-48 hours after intravenous administration of ${ }^{125} \mathrm{I}-\mathrm{mIBG}$ : an inhomogeneous distribution within individual tumours was found, with the most avid uptake in areas of viable undifferentiated neuroblasts. ${ }^{16} \mathrm{~A}$ study of the intracellular distribution of mIBG in human SK-N-SH neuroblastoma cell lines, xenografted into nude mice, by means of microautoradiography and secondary ion mass spectrometry microscopy, confirmed highly non-uniform distribution and, at an intracellular level, most of the radiopharmaceutical accumulated in the cytosol and perinuclear areas. ${ }^{17}$ These observations are of importance in selecting the most appropriate radionuclide emitter(s) for use in targeted treatment.

\section{Radiobiology}

Neuroblastoma is one of the most radiosensitive of all human tumours ${ }^{18}$ and external beam radiotherapy is employed with good benefit in the local control of disease, but the dose constraints of total body irradiation (TBI) preclude effective systemic radiotherapy. Even the combination of TBI with high dose chemotherapy and autologous bone marrow rescue has failed to show any benefit over high dose chemotherapy alone. ${ }^{19}$ If higher radiation doses could be delivered to the tumour while sparing normal tissues, radiotherapy has curative potential in this disease.

The radiobiological considerations that determine the efficacy of radioiodinated $\mathrm{mIBG}$ in neuroblastoma have also been both modelled and studied. ${ }^{20}$ Radiation induced cell death is generally assumed to result from lethal damage to the nucleus. As the cellular diameter of a neuroblast is $10-20 \mu \mathrm{m}$ and as the particulate emission 
from ${ }^{131} \mathrm{I}$ has a mean range of $800 \mu \mathrm{m}$, this will result in a 'crossfire' effect from adjacent cells that take up mIBG into cells that do not. This would be predicted to be advantageous in non-homogeneous tumours and, at the same time, potentially disadvantageous for example in the case of marrow infiltration where adjacent haematopoietic cells would be within range. ${ }^{125} \mathrm{I}$ emits Auger electrons with a range of $\sim 1 \mu \mathrm{m}$ and would therefore not exert any 'crossfire' effect, but ideally should be taken into the nucleus to exert maximum effect. ${ }^{125} \mathrm{I}$ mIBG has been used for therapy at the University of Michigan. ${ }^{22}$ An $\alpha$ emitting radionuclide would, in theory at least, be ideal. ${ }^{23}{ }^{211}$ At-metaastatobenzylguanidine has been synthesised and has been shown to induce in vitro cytotoxicity much more efficiently than ${ }^{131} \mathrm{I}-\mathrm{mIBG},{ }^{24}$ however there are major logistical problems related to production and safety that will probably preclude this agent for in vivo human treatment.

Another important variable in mIBG treatment is the specific activity of the radiopharmaceutical. Commercially available ${ }^{131} \mathrm{I}$-mIBG is of relatively low specific activity, that is the ratio of cold to radiolabelled molecules is high, and the cold molecules will compete for binding sites resulting in suboptimal tumour concentrations. Methods now exist for synthesis of high specific activity (or no carrier added) ${ }^{131}$ I-mIBG. ${ }^{25}$ The high specific activity preparation is be expected to enhance tumour uptake by a factor of around 10 as a result of improved target-to-background ratios, ${ }^{26}$ but large scale production is thought not be commercially viable at the present time.

Mathematical modelling predicts that ${ }^{131} \mathrm{I}-\mathrm{mIBG}$ would be most effective in smaller micrometastases. The ideal size is predicted to contain $\sim 10^{6}$ cells, with a lower cell kill in micrometastases smaller or larger than this. A study of spheroids of the human neuroblastoma cell line SK-H-BE (2c) of $250 \mu \mathrm{m}$ and $400 \mu \mathrm{m}$ size incubated in ${ }^{131} \mathrm{I}-\mathrm{mIBG}$ has shown that the larger size spheroids had a greater vulnerability as predicted by the model..$^{21}$ It may well be that targeted ${ }^{131}$ I-mIBG therapy can 'fill the gap' between chemotherapy, which is most effective in very small micrometastases, and external beam radiotherapy and surgery, which are more efficient in dealing with macroscopic tumours. These observations are also relevant to the sequencing of multimodal treatment and predict that targeted ${ }^{131}$ I-mIBG treatment should be employed earlier rather than later in relation to chemotherapy.

\section{Clinical studies}

Neuroblastoma remains the major therapeutic challenge in paediatric oncology and new treatment strategies are needed. Despite response rates of approximately $75 \%$ with modern dose intensive induction chemotherapy and high dose consolidation with stem cell rescue, no more than $25 \%$ of children over 1 year of age at diagnosis of metastatic neuroblastoma will be long term survivors. ${ }^{19}$

By 1991 reports had documented more than 250 children with advanced neuroblastoma who had received ${ }^{131}$ I-mIBG treatment. ${ }^{27}$ All of these treatments had been given to patients with chemoresistant disease either after induction chemotherapy or at relapse. Although the indications for treatment, methods of administration and criteria for assessment of response were variable, three of these earlier studies will be singled out:

(1) A phase II study from the Netherlands Cancer Institute reported on 53 patients with progressive or recurrent disease in whom conventional treatment had failed. ${ }^{28}$ Empirical therapeutic activities of 3.7-7.4 GBq were given and some received more than one treatment. The results showed complete responses in $7 / 53$ patients (13\%), partial responses ( $>50 \%$ reduction in tumour volume) in $23 / 53$ $(43 \%)$, and stable disease in a further 10 patients $(19 \%)$.
(2) The German Neuroblastoma Trial published results on 47 children who progressed or relapsed after treatment with standard chemotherapy. ${ }^{29}$ Patients received 1-6 courses of ${ }^{131} \mathrm{I}-\mathrm{mIBG}$ (mean total activity $14.4 \mathrm{GBq}$ ). The results showed that $9 / 47$ patients $(19 \%)$ achieved a complete or very good partial response, and a partial response was seen in a further 13 patients $(28 \%)$.

(3) The prospective United Kingdom Children's Cancer Study Group (UKCCSG) phase I/II study, which was a dose finding and toxicity study of ${ }^{131} \mathrm{I}-\mathrm{mIBG}$ therapy in stage 4 patients with a poor response to chemotherapy, reported partial responses in $8 / 24$ patients $(33 \%))^{30}$

These three studies demonstrate that $\mathrm{mIBG}$ has single agent efficacy comparable with that of the chemotherapy drugs presently employed in induction protocols.

Two studies have reported the feasibility of administering the maximum tolerated activity of ${ }^{131} \mathrm{I}-\mathrm{mIBG}$ in combination with high dose chemotherapy and stem cell rescue as consolidation treatment. One study used ${ }^{131} \mathrm{I}-\mathrm{mIBG}$ (average activity $11.1 \mathrm{GBq}$ ) followed by high dose carboplatin and melphalan, ${ }^{31}$ while the other incorporated total body irradiation and no additive extramedullary toxicities were encountered. ${ }^{32}$

The dose limiting toxicity of ${ }^{131} \mathrm{I}-\mathrm{mIBG}$ when administered after intensive chemotherapy is thrombocytopenia. ${ }^{30}$ Risk factors for the development of severe thrombocytopenia in this setting include bone marrow infiltration, prior intensive chemotherapy, and renal impairment. ${ }^{33}$

An important single centre study of $\mathrm{mIBG}$ as first line treatment for high risk neuroblastoma was started in Amsterdam in $1991 .^{34}$ An interim report describes the first 31 children with inoperable neuroblastoma treated with a minimum of two cycles of ${ }^{131} \mathrm{I}$-mIBG $(7.4 \mathrm{GBq}$ followed by 3.7 GBq). Thereafter, operability was assessed and, if the primary tumour was deemed unresectable, further fractions of $3.7 \mathrm{GBq}$ of ${ }^{131} \mathrm{I}-\mathrm{mIBG}$ were administered. Chemotherapy was reserved for those who failed to become operable after repeated ${ }^{131}$ I-mIBG treatments $(7 / 31)$ and for those with residual disease after surgical resection of the primary tumour. Isolated depression of the platelet count was seen in only 11 patients, while two patients developed pancytopenia. Severe thrombocytopenia was not encountered despite extensive bone marrow infiltration in some of these patients. The treatment was extremely well tolerated with virtually no sickness, no hair loss, and a mean weight gain of $9.5 \%$ between diagnosis and surgery. Eighty one per cent responded at metastatic sites and $73 \%$ responded at the primary site. Only $8 / 31$ had an inadequate response to ${ }^{131} \mathrm{I}$-mIBG treatment and went onto chemotherapy. At surgery $16 / 31$ had a $>95 \%$ resection and $3 / 31$ a partial resection of their tumour. ${ }^{35}$

\section{Future strategies}

The radiobiological considerations predict that the combination of mIBG and chemotherapy administered as initial treatment for neuroblastoma would be the optimum clinical strategy. ${ }^{20}$ At least in vitro, synergy can be demonstrated between external beam radiation treatment and platinum derivatives. ${ }^{36}$ Pretreatment with cisplatin might enhance mIBG uptake into neuroblasts. ${ }^{15}$ The feasibility of treating children with a mIBG/cisplatin combination has been reported in relapsed neuroblastoma, ${ }^{37}$ but the optimum sequence and dose in newly diagnosed patients has still to be determined.

The UKCCSG is engaged in a multicentre pilot phase I study in poor prognosis newly diagnosed patients. This is essentially a feasibility and dose finding study of mIBG administered as initial treatment five days before the standard OPEC/OJEC chemotherapy protocol. The aim is 
to perform whole body dosimetry at the time of the diagnostic ${ }^{123} \mathrm{I}-\mathrm{mIBG}$ scan in order to calculate the activity of ${ }^{131} \mathrm{I}-\mathrm{mIBG}$ that will deliver the required whole body dose. The logistics of delivering targeted radiotherapy within two weeks of diagnosis in young children, who are systemically ill and need to be nursed in a radiation protected environment with limited parental contact for several days, are considerable but can be surmounted by having the radiation facility within the paediatric oncology ward. The principle is to employ the maximum tolerated dose of ${ }^{131} \mathrm{I}-$ mIBG and the haematological and any other toxicity of a starting whole body dose of $1.5 \mathrm{~Gy}$ will be determined before dose escalation. The aim is to set up a national randomised trial in stage 4 neuroblastoma, possibly in collaboration with other European groups, to compare the remission rate induced by the current most effective chemotherapy with that of combined treatment.

\section{Conclusions}

Although it is more than 10 years since mIBG was first used to treat neuroblastoma, there is still some way to go before the therapy is optimised. There is considerable potential for this modality to improve the results of treatment of advanced stage neuroblastoma, but it is too soon to know which of the new strategies employing mIBG will be the most effective in achieving sustained remissions. It is clear that the short term toxicity is considerably less than that of current chemotherapy regimens, but there is a need to be vigilant for the possible long term toxicities of ${ }^{131} \mathrm{I}$-mIBG treatment and, although there are few long term survivors to date, there is already one report of late thyroid dysfunction. ${ }^{38}$ There is also a theoretical risk of radiation damage to the bladder wall, radiation induced secondary malignancy, and adrenal insufficiency consequent to high dose targeted radioiodine treatment.

SIMON MELLER

Children's Department,

Royal Marsden NHS Trust,

Downs Road, Sutton,

Surrey SM2 5PT

1 Order SE. Systemic radiotherapy: the new frontier. Int $\mathcal{F}$ Radiat Oncol Biol Phys 1990;18: 981-92.

2 Beierwaltes WH, Nishiyama RH, Thompson NW, Copp JE, Kubo A Survival time and 'cure' in papillary and follicular thyroid carcinoma with distant metastases: statistics following University of Michigan therapy. $\mathcal{F}$ Nucl Med 1982;23:561-8.

3 Kohler G, Milstein C. Continuous cultures of fused cells secreting antibody of predefined specificity. Nature 1975;256:495-7.

4 Lashford LS, Clark J, Kemshead JT. Systemic administration of radionuclides in neuroblastoma as planned radiotherapeutic intervention. Med Pediatr Oncol 1990;18:30-6.

5 Cheung NK, Lazarus H, Miraldi FD. Ganglioside GD2 specific monoclonal antibody 3F8: a phase I study in patients with neuroblastoma and malignant melanoma. $\mathcal{F}$ Clin Oncol 1978;5:1430-40.

6 Pizer B, Papanastassiou V, Hancock J, Cassano W, Coakham H, Kemshead J. A pilot study of monoclonal antibody targeted radiotherapy in the treatJ. A pilot study of monoclonal antibody targeted radiotherapy in the treat-
ment of central nervous system leukaemia in children. Br f Haematol 1991; ment of cen

7 Wieland DM, Swanson DP, Brown LE, Beierwaltes WH. Imaging the adrenal medulla with an ${ }^{131}$ I-labeled anti-adrenergic agent. $₹$ Nucl Med 1979;20: nal me

8 Sisson JC. Frager MS, Valk TW, et al. Scintigraphic localization of phaeochromocytoma. N Engl f Med 1981;305:12-7.

9 Kimmig B, Brandeis WE, Eisenhut M, Bubeck B, Hermann HJ, zum Winkel K. Scintigraphy of a neuroblastoma with I-131-metaiodobenzylguanidine. $\mathcal{f}$ Nucl Med 1984;25:773-5.

10 Wafelman AR, Hoefnagel CA, Maes RA, Beijnen JH. Radioiodinated metaiodobenzylguanidine: a review of its biodistribution and pharmacokinetics, drug interactions, cytotoxicity and dosimetry. Eur 7 Nucl Med 1994; 21:545-59.

11 Tobes MC, Jaques S Jr, Wieland DM, Sisson JC. Effect of uptake-one inhibitors on the uptake of norepinephrine and metaiodobenzylguanidine. f Nucl Med 1985;26:897-907.
12 Smets LA, Loesberg C, Janssen M, Metwally EA, Huiskamp R. Active uptake and extravesicular storage of $\mathrm{m}$-iodobenzylguanidine in human neuroblastoma SK-N-SH cells. Cancer Res 1989;49:2941-4.

13 Smets LA, Janssen M, Rutgers M, Ritzen K, Buitenhuis C. Pharmacokinetics and intracellular distribution of the tumor-targeted radiopharmaceutical m-iodobenzylguanidine in SK-N-SH neuroblastoma and PC-12 phaeochromocytoma cells. Int f Cancer 1991;48:609-15.

14 Montaldo PG, Carbone R, Ponzoni M, Cornaglia-Ferraris G. Gammainterferon increases metaiodobenzylguanidine incorporation and retention in human neuroblastoma cells. Cancer Res 1992;52:4960-4.

15 Armour A, Cunningham SH, Gaze MN, Wheldon TE, Mairs RJ. The effect of cisplatin pre-treatment on the accumulation of $\mathrm{mIBG}$ by neuroblastoma cells in vitro. Br ₹ Cancer 1997;75:470-6.

16 Moyes JSE, Babich JW, Carter R, Meller ST, Agrawal M, McElwain TJ. Quantitative study of radioiodinated metaiodobenzylguanidine uptake in children with neuroblastoma: correlation with tumour histopathology. $f$ Nucl Med 1989;30:474-80.

17 Clerc J, Halpern S, Fourre C, et al. SIMS microscopy imaging of the intratumor biodistribution of metaiodobenzylguanidine in the human SK$\mathrm{N}-\mathrm{SH}$ neuroblastoma cell line xenografted in to nude mice. $7 \mathrm{Nucl} \mathrm{Med}$ 1993;34:1565-70.

18 Deacon JM, Wilson PA, Peckham MJ. The radiobiology of human neuroblastoma. Radiother Oncol 1985;3:201-9.

19 Ladenstein R, Lasset C, Hartmann O, et al. Impact of megatherapy on survival after relapse from stage 4 neuroblastoma in patients over 1 year of age at diagnosis: a report from the European Group for Bone Marrow Transplantation. $\mathcal{F}$ Clin Oncol 1993;11:2330-41.

20 O'Donoghue JA, Wheldon TE, Babich JW, Moyes JSE, Barrett A, Meller ST. Implications of the uptake of ${ }^{131}$-radiolabelled metaiodobenzylguanidine (mlBG) for the targeted radiotherapy of neuroblastoma. $\mathrm{Br} \mathcal{F}$ Radiol 1991;64:428-34.

21 Gaze MN, Mairs RJ, Boyack SM, Wheldon TE, Barrett A. ${ }^{131} \mathrm{I}$ meta-iodobenzylguanidine therapy in neuroblastoma spheroids of different sizes. Br 7 Cancer 1992;66:1048-52.

22 Sisson JC, Hutchinson RJ, Shapiro B, et al. Iodine-125-MIBG to treat neuroblastoma: preliminary report. F Nucl Med 1990;31:1479-85.

23 Vaidyanathan G, Zalutsky MR. Targeted therapy using alpha emitters. Phys Med Biol 1996;41:1915-31.

24 Strickland DK, Vaidyanathan G, Zalutsky MR. Cytotoxicity of alphaparticle-emitting $\mathrm{m}$-[211At] astatobenzylguanidine on human neuroblastoma cells. Cancer Res 1994;54:5414-9.

25 Gaze MN, Mairs RJ, Vaidyanathan G, Zalutsky MR. Synthesis of carrier-free ${ }^{131} \mathrm{I}$-meta-iodobenzyl-guanidine by novel routes to enhance therapeutic efficiency in neuroblastoma. Prog Clin Biol Res 1994;385:34753.

26 Mairs RJ, Russell J, Cunningham S, et al. Enhanced tumour uptake and in vitro radiotoxicity of no-carrier-added [131I] meta-iodobenzylguanidine: implications for the targeted radiotherapy of neuroblastoma. Eur 7 Cancer 1995;31A:576-81.

27 Troncone L, Galli G. Proceedings of the international workshop on the role of [131I] metaiodobenzylguanidine in the treatment of neural crest tumours. F Nucl Biol Med 1991;35:177-362.

28 Voute PA, Hoefnagel CA, de Kraker J, Valdes Olmos R, Bakker DJ, van de Kleij AJ. Results of treatment with ${ }^{131} \mathrm{I}$-metaiodobenzylguanidine in patients with neuroblastoma. Future prospects of zetotherapy. In: Evans patients with neuroblastoma. Future prospects of zetotherapy. In: Evans research 3. New York: Wiley-Liss, 1991: 439-45.

29 Klingebiel T, Berthold F, Treuner J, et al. Metaiodobenzylguanidine (mIBG) in the treatment of 47 patients with neuroblastoma: results of the German Neuroblastoma Trial. Med Pediatr Oncol 1991;19:84-8.

30 Lashford LS, Lewis IJ, Fielding SL, et al. Phase I/II study of iodine 131 metaiodobenzylguanidine in chemoresistant neuroblastoma: a United Kingdom Children's Cancer Study Group investigation. F Clin Oncol 1992; 10:1889-96.

31 Corbett R, Pinkerton CR, Tait D, Meller S. [131I] metaiodobenzylguanidine and high-dose chemotherapy with bone marrow rescue in advanced neuroblastoma. F Nucl Biol Med 1991;35:228-31.

32 Gaze MN, Wheldon TE, O'Donoghue JA, Hilditch TE, McNee SG, Barrett A. Multi-modality megatherapy with [131I] meta-iodobenzylguanidine, high dose melphalan and total body irradiation with bone marrow rescue: feasibility study of a new strategy for advanced neuroblastoma. Eur $7 \mathrm{Can}$ cer 1995;31A:252-6.

33 Sisson JC, Hutchison RJ, Carey JE, et al. Toxicity from the treatment of neuroblastoma with ${ }^{131}$ I-meta-iodobenzylguanidine. Eur F Nucl Med 1988;14: $337-40$.

34 Hoefnagel CA, de Kraker J, Voute PA, Valdes Olmos RA. Preoperative I-131 $\mathrm{mIBG}$ therapy of neuroblastoma at diagnosis ('mIBG de novo'). $7 \mathrm{Nucl} \mathrm{Biol}$ Med 1991;35:248-51.

35 de Kraker J, Hoefnagel CA, Caron H, et al. First line targeted radiotherapy, a new concept in the treatment of advanced stage neuroblastoma. Eur $\mathcal{F}$ Cancer 1995;31A:600-2.

36 Muggia FM, Glatstein E. Summary of investigations on platinum compounds and radiation interactions. Int $\mathcal{F}$ Radiat Oncol Biol Phys 1979;5: 1407-10.

37 Mastrangelo R, Tornesello A, Riccardi R, et al. A new approach in the treatment of stage IV neuroblastoma using a combination of 131-Imetaiodobenzylguanidine (mIBG) and cisplatin. Eur $\mathcal{F}$ Cancer 1995;31A: 606-11.

38 Picco P, Garavanta A, Claudiani F, Gattorno M, De Bernardi B, Borrone C. treatment for children with neuroblastoma. Cancer 1995;76:1662-4. 